\title{
Las cooperativas ante un nuevo ciclo de transformaciones. Su contribución a los retos del tejido productivo vasco*
}

\author{
Cooperatives in the light of a new cycle of transformations. \\ Their contribution to the challenges facing the Basque \\ productive framework
}

Igor Ortega Sunsundegui

Investigador en LANKI, Instituto de estudios cooperativos de MONDRAGON UNIBERTSITATEA

DOI: $10.1387 /$ reves.20516

Fecha de entrada: 10/06/2018

Fecha de aceptación: 25/09/2018

Sumario: 1. Introducción.-2. La economía vasca ante un nuevo ciclo de transformaciones.-3. La contribución de las cooperativas a los retos del tejido productivo: principales resultados. 3.1. La Economía Social Vasca se encuentra inmersa en un importante proceso de recomposición interna: Las cooperativas aumentan su peso relativo en el seno de las familias más representativas de la Economía Social. 3.2. La mayor capacidad de resiliencia de las cooperativas: las cooperativas manifiestan un mejor comportamiento en cuanto al mantenimiento de la actividad y el empleo en contextos de crisis. 3.3. El reto de la industria avanzada: la contribución de las cooperativas es más significativa en los segmentos estratégicos y tractores de la economía. 3.4. Las cooperativas demuestran una mayor orientación innovadora que el resto de empresas.-4. Conclusiones.-5. Bibliografía.

\section{Resumen}

La economía vasca y el tejido empresarial del territorio enfrentan un contexto de grandes transformaciones caracterizado por la eclosión de las economías emergentes y por la apuesta de las economías avanzadas por la industria de alta tec-

* El presente artículo se basa en el proyecto de investigación Aportación de las cooperativas a los retos del tejido productivo vasco realizado por LANKI, Instituto de Estudios Cooperativos de MONDRAGON UNIBERTSITATEA, en colaboración con la DIPUTACIÓN FORAL DE GIPUZKOA en el marco del programa ETORKIZUNA ERAIKIZ. 
nología y los sistemas regionales de innovación. En el presente artículo, utilizando diversas fuentes estadísticas, se analiza la contribución que en este escenario realizan las cooperativas en relación al resto de modelos de empresa. El análisis identifica el importante rol que asumen las cooperativas no solo en cuanto a su capacidad de resiliencia sino también en cuanto a coliderar el proceso de transición de la economía vasca hacia un modelo de desarrollo sostenido sobre la base de un tejido industrial innovador y tecnológicamente avanzado.

\title{
Palabras clave:
}

Cooperativas, Aportación social, tejido productivo, industria.

\begin{abstract}
The Basque economy and business fabric in the territory are facing a context rife with huge transformations, characterised by the dawn of emerging economies and the focus of high-technology industry and regional innovation systems on advanced economies. Using different statistical sources, this article analyses the contribution made by cooperatives on this stage compared to the rest of company models. The analysis identifies the important role of cooperatives, not only as far as their resilience is concerned, but also how they co-lead the transition process for the Basque economy toward a sustainable development model, built on the foundation of an innovative, technologically advanced industrial fabric.
\end{abstract}

\section{Keywords:}

Cooperatives, social contribution, production fabric, industry.

\section{Claves Econlit:}

P130, L600, M140, 0140.

\section{Introducción}

El surgimiento y desarrollo de un proyecto cooperativo no es posible concebirlo sin su intrínseca conexión con las necesidades y los retos de la sociedad de la cual se forma parte. «Identificar las necesidades sociales y abordarlas...» es una de las máximas éticas que impulsaron a la acción a los fundadores de las primeras cooperativas en Mondragón. En este sentido, las cooperativas se conciben como herramientas que, inspiradas por un sentido profundo de la justicia social, tratan de responder a los retos sociales percibidos por sus protagonistas. La fase moderna del cooperativismo vasco, aquella que se inicia a finales de la década de los 50 del siglo $\mathrm{xx}$, surge de este impulso ético y transformador.

Siguiendo la estela de los textos de los fundadores (Azurmendi, 1984; Larrañaga, 1998; Ormaetxea 1999 y 2003) se puede sintetizar en cuatro las as- 
piraciones básicas que se encuentran en la génesis de la experiencia de Mondragón: La contribución al desarrollo económico y al bienestar material de la sociedad vasca a través de la promoción de cooperativas principalmente industriales; la contribución al desarrollo de una sociedad justa y equitativa mediante la implementación de criterios y mecanismos de solidaridad interna y externa; el desarrollo de un modelo de empresa democrático, participativo y sustentando en el desarrollo de las capacidades humanas a través de la cooperación; y la contribución al desarrollo de un nuevo orden social sustentado en los valores de la cooperación, la democracia, la solidaridad y el compromiso social.

Pero del conjunto de estas aspiraciones, la autorresponsabilidad asumida en el desarrollo económico del País Vasco ha sido uno de los rasgos más singulares que caracterizan los orígenes del moderno cooperativismo vasco. Ante la despreocupación por las innovaciones técnicas y el desarrollo tecnológico de las empresas por parte de la clase empresarial de la época, mediante la promoción y la difusión de experiencias cooperativas, se aspiraba, entre otros objetivos, a dotar de herramientas que posibilitaran la generación de riqueza y el desarrollo de las bases materiales para el bienestar de las comunidades. Es por esta razón que desde los inicios se hace una apuesta clara por el sector industrial y por dotar de contenido tecnológico a las empresas cooperativas. La promoción y la expansión de cooperativas de trabajo industriales fue la vía adoptada para que la clase trabajadora tomara la iniciativa por el desarrollo de un modelo económico pujante, capaz de mantener el ritmo de desarrollo de las economías avanzadas, y poder contribuir así a "hacer país», propósito que comenzaba por la construcción de una "economía vigorosa y humana» (Ormaetxea, 2003).

Pensamos que es desde el decidido impulso de estas aspiraciones como se puede llegar a explicar el proceso de extensión y expansión que ha hecho mundialmente singular a esta experiencia cooperativa, y la importante contribución que ha realizado el cooperativismo vasco al desarrollo económico y social de nuestro territorio.

Hoy, unos 60 años después del inicio de las primeras experiencias cooperativas, se puede afirmar que la economía vasca se encuentra ante un nuevo estadio de bifurcación. En este contexto, y en perspectiva de los retos que enfrenta la sociedad vasca en este nuevo ciclo de transformaciones económicas, es donde nos hemos propuesto indagar sobre la aportación que realizan las cooperativas a los retos que enfrenta el tejido productivo vasco en el nuevo escenario económico que parece abrirse. En el objetivo de sustentar el análisis en resultados tangibles y cuantificables hemos recurrido a diferentes fuentes estadísticas que aportan información sobre el estado y la evolución de variables significativas de la realidad cooperativa (Los informes bianuales de Estadísticas de la Economía Social del Departamento de Empleo y Políticas Sociales del Gobierno Vasco y el Informe de Situación de la Economía Social Vasca del OVES). Además, con el fin de 
determinar la contribución específica que realizan las cooperativas respecto a los modelos de empresa mercantiles se ha complementado el análisis estadístico con diferentes indicadores disponibles en las fuentes del Eustat.

Por lo tanto, ¿Cuál es la aportación que realizan las cooperativas a los retos que enfrenta el tejido productivo vasco en el contexto actual? A continuación presentamos las principales resultados del análisis.

\section{La economía vasca ante un nuevo ciclo de transformaciones}

La economía vasca y el tejido empresarial del territorio se enfrentan a un escenario de grandes transformaciones provocado por la eclosión de las economías emergentes y, en concreto, por la formidable expansión que se espera alcancen las economías India y China (Prospektiker, 2012). Lo relevante de la evolución de estos países es que su capacidad competitiva dejará de sustentarse únicamente en el coste para hacerlo también en ámbitos tan significativos como las marcas, la innovación y el desarrollo tecnológico, sin obviar los diferenciales que emanarán de la dimensión de muchas de estas empresas (Astigarraga, 2016; Ugarte, 2013).

La irrupción de nuevos países emergentes como agentes económicos de primer orden está provocando ya una reestructuración en las posiciones relativas de los principales actores de la economía mundial (Navarro y Sabalza, 2016). En este contexto destaca la especial encrucijada en la que se encuentra la economía europea que ve cómo va perdiendo peso en la economía mundial (Letta, 2017; Prospektiker, 2012), manifiesta dificultades para seguir el ritmo de desarrollo de economías avanzadas como la de EEUU (García Delgado y Myro, 2016) , y en la que potencias emergentes como China la empiezan a alcanzar en ámbitos tan significativos como el esfuerzo inversor en I+D (Molinas y Ramirez, 2017; García Quevedo, 2016; Sudrià y Garrido, 2016). Como respuesta a este relativo declive, y en el contexto de la nueva revolución tecnológica (Ortega, 2016; Pérez, 2010), estamos asistiendo a una reorientación de la política económica de la Unión Europea y la de sus principales países sustentada en la priorización de la industria avanzada de alta tecnología (Duch y Blanco, 2016; García Quevedo, 2016) y la apuesta por unas economías intensas en innovación a través del desarrollo de los sistemas locales de innovación (Orkestra, 2015; Zurbano, 2015).

La economía vasca no se encuentra al margen del probable impacto de estas tendencias. A priori, la economía vasca demuestra una serie de fortalezas a la hora de encarar este escenario: su relativa especialización industrial que, a pesar de su relativa pérdida de peso en el conjunto de la economía, la sitúa entre el grupo de países que mantienen una industria manufacturera relativamente fuerte (Navarro y Sabalza, 2016); el importante desarrollo de la infraestruc- 
tura innovadora y la pluralidad de agentes que configuran el sistema vasco de innovación que posicionan a la CAPV entre el grupo de regiones de innovación alta (Navarro, 2016; Orkestra, 2017); el margen disponible para el desarrollo de políticas propias y estrategias adecuadas a la realidad del territorio gracias a la autonomía y la densidad de su entramado institucional (Orkestra, 2015; Zurbano, 2016); e, incluso, el capital social y de confianza acumulado en el territorio que potencialmente permiten un mayor nivel y calidad del flujo de conocimiento entre los agentes implicados en el sistema local de innovación (Zurbano, 2016). Todas ellas son factores diferenciales en el contexto del desarrollo de las economías de la innovación y el conocimiento.

Sobre estas bases el territorio vasco ha sido capaz de desarrollar una economía próspera, competitiva y de un importante valor añadido que, al contrario del modelo productivo español, ha permitido una mayor especialización en sectores de altos salarios sin merma de productividad (Orkestra, 2017).

Pero, el nuevo escenario que venimos analizando, introduce una serie de incertidumbres sobre la capacidad de adaptación del tejido productivo vasco a las nuevas condiciones de la globalización. La comparativa con las regiones europeas más avanzadas también indican una serie de potenciales debilidades de la economía vasca: la práctica inexistencia de grandes multinacionales y conglomerados industriales que en el resto de regiones demuestran tener un efecto tractor sobre el conjunto de la industria (CES, 2015); una menor proporción de empresas grandes en el territorio y en la que, además, las grandes pymes vascas son de dimensión menor que sus competidores de referencia (Orkestra, 2015); la predominancia en el tejido productivo vasco de empresas que operan en sectores tecnológicos bajo y medio-bajo (Borja, 2012; Zelaia, 2015) y, por lo tanto, más expuestas a la copia rápida de países emergentes con capacidad de producirlas a un menor coste (Ugarte, 2013); y la realidad de un territorio, el espacio económico vasco, más pequeño que a priori puede determinar la existencia de unas capacidades (humanas y monetarias) más limitadas para poder acometer determinados proyectos de inversión óptima (CES, 2015).

En este nuevo escenario son varios los factores que desde el punto de vista del tejido productivo vasco merecen prestar una especial atención. Por un lado, la mayor competencia de las empresas de los países emergentes (tanto europeos como asiáticos) que gracias al desarrollo de las capacidades tecnológicas de sus empresas son ya capaces de competir con menores costes y con niveles de calidad similar al estándar medio de las empresas europeas y vascas. Las empresas industriales tradicionales con una composición tecnológica media o baja son más vulnerables a la competencia internacional en este escenario de la globalización 2.0.

Por otro, la capacidad de la economía vasca para seguir el ritmo de desarrollo de las regiones europeas más dinámicas. Los países industrializados avanzados es- 
tán apostando decididamente por la industria de alta tecnología y los servicios intensivos en conocimiento en sus estrategias de diferenciación competitiva frente a los países emergentes. En estas nuevas claves, el esfuerzo tecnológico, el volumen de recursos destinados a la $\mathrm{I}+\mathrm{D}$, junto con el conjunto de medidas que favorezcan la innovación y la eficiencia de las empresas, son considerados como los principales factores competitivos de las empresas y de los territorios.

A este escenario de incertidumbres se le añade la posibilidad de irrupción de modelos de negocio disruptivos que erosionen la posición de alguna de las actividades principales de la economía de la CAPV (Kamp, 2016; Navarro y Sabalza, 2016); o el peligro de desterritorialización de importantes empresas sea por decisiones de deslocalización o por apropiaciones y absorciones por capitales financieros (B+I Strategy, 2016).

En este contexto de transformaciones, ¿cómo se está posicionando la economía vasca? Sabemos, por las investigaciones especializadas que abordan el tema, que la actividad económica y sobre todo la dinámica innovadora de los países tienden a concentrarse en el espacio. Ello tiene importantes implicaciones para el análisis comparado de la realidad vasca. Una comparativa con los promedios de los diferentes estados puede dar lugar a una imagen «embellecida» de la economía vasca. Más aún, si esta comparación se realiza con los promedios de España o la realidad de sus comunidades autónomas (Navarro, 2010: 152). Esto podría dar lugar a una lectura autocomplaciente de la economía de la CAPV, y distorsionar la posición real de la CAPV respecto a las regiones de referencia. En este sentido, una perspectiva que aborde la realidad regional de los países ofrece una información más valiosa que el uso de los promedios estatales ${ }^{1}$.

1 Para elaborar el apartado nos hemos basado en informes de diferentes instituciones que analizan la salud competitiva y el dinamismo del tejido productivo vasco en relación a las regiones europeas. Los Informes de Competitividad de Orkestra (2015 y 2017) comparan los indicadores de la CAPV con: a) El conjunto de las 272 regiones europeas; b) un grupo de 30 regiones europeas de referencia, escogidas por ser las que representan una mayor similitud con la CAPV en términos de condiciones estructurales de partida (sociodemográficas, de especialización económica y tecnológica, y de estructura empresarial); c) las comunidades autónomas del Estado Español; d) complementariamente en pos de una versión más actualizada de los indicadores, con la media europea, España, Alemania (como uno de los países más avanzados) y la República Checa (escogida por ser uno de los países emergentes que destacan entre los países de la ampliación europea por su nivel de renta y por el resto de indicadores de competitividad y que, como país industrial, puede resultar un competidor de la CAPV. El informe del CES Vasco (2015), por su parte, presenta un estudio comparado de la economía vasca con tres regiones europeas (Baden-Württemberg en Alemania, Flandes en Bélgica y Emilia-Romagna en Italia) seleccionadas por compartir una serie de características estructurales similares a la CAPV: altos niveles de bienestar económico, fuerte peso del sector industrial, orientación a la innovación y a la $\mathrm{I}+\mathrm{D}$, un elevado grado de apertura al exterior, y amplios niveles de autonomía política que les permiten desarrollar políticas propias muy activas en la promoción económica, productiva y empresarial. 
El análisis comparado de la dinámica del tejido productivo del país con las regiones europeas, nos deja un panorama de claroscuros:

— El impacto de la crisis en Euskadi, medido en términos de reducción del PIB per cápita, exportaciones, destrucción de empleo o en los indicadores de satisfacción de vida, ha sido mayor que en las regiones más dinámicas de Europa. Además, la economía vasca ha manifestado una mayor lentitud en dar señales de recuperación.

- A pesar de ello, recuperada la senda del crecimiento, la economía vasca muestra de nuevo señales de su fortaleza con niveles de crecimiento superiores a las principales economías de Europa en el periodo 2015-16. La CAPV, en términos de PIB y renta per cápita, se sitúa entre las regiones más prósperas de Europa, manifestando también un buen comportamiento en cuanto a la productividad y las exportaciones de su industria.

- La menor ausencia de multinacionales o grandes empresas parece compensarse con el dinamismo que reflejan las pymes medianas vascas. El ratio de 14 «campeones ocultos» por millón de habitantes que presenta la CAPV es reflejo de esta realidad. Este ratio es muy superior a la de países como Francia, EEBB o Japón (1-2 por millón de habitantes), se encuentra por encima de los Países Bajos (10) y en la línea de países económicamente avanzados como Suiza, Austria y Alemania (14-16), aunque es menor que los ratios que presentan los Lander Baden-Wunttenberg o Hamburgo (25-29 campeones ocultos por millón de habitantes).

- Las principales señales de incertidumbre se asocian la actividad innovadora. Tras el periodo de la crisis, la economía vasca demuestra dificultades para mantener el ritmo de desarrollo e innovación de las regiones europeas más dinámicas. Esta realidad se está viendo reflejada en la evolución negativa de los principales indicadores relativos a la innovación tales como el porcentaje del Gasto en $\mathrm{I}+\mathrm{D}$ respecto al PIB, el porcentaje de gasto en actividades de I+D en relación a la facturación total destinado por las empresas, la evolución del número de empresas que realizan acciones de $\mathrm{I}+\mathrm{D}$, o la incorporación de innovaciones tecnológicas y no tecnológicas por parte de las empresas vascas. La evolución negativa del esfuerzo en I+D se está empezando a reflejar en una merma de la posición relativa de la economía vasca en los principales indicadores europeos.

- Las dificultades se observan también en la traslación de los buenos resultados económicos a los indicadores relacionados con el progreso social del país. Los análisis comparados muestran que el nivel relativo del progreso social o bienestar no se corresponde con el nivel del PIB per cápita de la CAPV: mientras la CAPV ocupa la posición 35 de en- 
tre las 272 regiones en cuanto al PIB per cápita, solo se encuentra en la posición 102 en el Índice de Progreso Social Europeo.

En suma, la fortaleza de la economía vasca parece haberse sustentado en la relativa especialización industrial de su tejido productivo, y en el esfuerzo realizado por las principales empresas en el desarrollo tecnológico de sus productos y la mejora de la calidad y la eficiencia de sus procesos de fabricación. Sobre esta base el tejido empresarial vasco ha demostrado una gran capacidad de adaptación a las condiciones competitivas impuestas por una economía en proceso de globalización. Las dudas residen en si estas bases resultarán suficientes para enfrentar los retos del nuevo escenario.

En la industria vasca predominan las actividades manufactureras tradicionales con una fuerte presencia de sectores con niveles tecnológicos medio-bajo y bajo. Por lo tanto, una parte importante de nuestro tejido productivo puede verse expuesta a la mayor presión competitiva de empresas emergentes con capacidades tecnológicas y de calidades similares pero con menores costes de producción. A su vez, el esfuerzo inversor de las empresas innovadoras vascas parece principalmente orientada hacia la mejora de productos y procesos, con poca incidencia de las innovaciones organizativas o comerciales. Todo ello puede indicar una relativa fortaleza en el desarrollo de productos existentes, pero una debilidad en cuanto a la capacidad de incorporar al mercado nuevos productos o servicios, y el desarrollo de nuevos modelos de negocio en un contexto que también se va a caracterizar por la irrupción de nuevas actividades disruptivas.

No hay duda de que uno de los principales retos que enfrenta el tejido productivo vasco en el nuevo ciclo económico que empieza a abrirse, pasa por el desarrollo de una estrategia de diferenciación, sostenida en un incremento del nivel tecnológico y las capacidades innovadoras de las empresas, que permita una mayor especialización en actividades de mayor valor añadido. Asimismo, de la capacidad que demuestre la economía vasca para transitar de una manera prudencialmente ágil y rápida desde los sectores en declive o vulnerables a la competencia de los productos de bajo precio procedente de las empresas con menores costes de producción, hacia actividades de mayor valor añadido, dependerá en gran medida la posibilidad de extender las bases del bienestar al conjunto de la población vasca.

En este sentido, la ralentización del esfuerzo inversor que parece inferirse de los principales indicadores relativos a la $\mathrm{I}+\mathrm{D}$ de la economía vasca es un dato preocupante que genera incertidumbre sobre la capacidad competitiva futura de las empresas del territorio. Esto, a su vez, dada las diferentes posiciones, capacidades y ritmos que se perciben en los procesos de transición entre las empresas vascas, acrecienta el riesgo de avanzar por la senda de un desarrollo dual, en la que la pertenencia a diferentes empresas podría derivar en un incremento de las desigualdades sociales del territorio. 


\section{La contribución de las cooperativas a los retos del tejido productivo: principales resultados}

¿Cuál es la aportación que realizan las cooperativas a los retos que enfrenta el tejido productivo vasco en el contexto actual? A continuación presentamos las principales resultados del análisis.

\subsection{La Economía Social Vasca se encuentra inmersa en un importante proceso} de recomposición interna: Las cooperativas aumentan su peso relativo en el seno de las familias más representativas de la Economía Social

El sector de la economía social en Euskadi, con datos del 2014, representa el $15,7 \%$ de las entidades y empresas, y el 8,3\% del empleo del conjunto de la CAPV. Las cooperativas se pueden considerar como la principal familia del heterogéneo espectro de la economía social vasca. No, quizá, en cuanto al número de entidades, ya que las asociaciones y las fundaciones con el $90 \%$ son el grupo más representativo en la economía social de Euskadi, pero sí en cuanto a volumen de empleo (donde el 6\% de las cooperativas constituyen el 65\% del empleo del conjunto de la economía social) y en cuanto al peso relativo de las cooperativas en relación a las principales variables económicas.

\section{Tabla 1}

Peso relativo de las cooperativas en relación a la economía social vasca. 2014

\begin{tabular}{l|rc|rr}
\hline \multirow{2}{*}{} & \multicolumn{2}{|c|}{ Entidades } & \multicolumn{2}{c}{ Empleo } \\
\cline { 2 - 5 } & ABS. & $\%$ VER. & ABS. & $\%$ VER. \\
\hline Cooperativas & 1.533 & $6,29 \%$ & 55.958 & $65,47 \%$ \\
Sociedades laborales & 709 & $2,90 \%$ & 6.679 & $7,81 \%$ \\
C. especiales de empleo & 9 & $0,03 \%$ & 7.746 & $9,06 \%$ \\
Empresas de inserción & 45 & $0,18 \%$ & 575 & $0,67 \%$ \\
S. agrarias de transf. & 101 & $0,41 \%$ & 239 & $0,27 \%$ \\
Cofradías pescadores & 16 & $0,06 \%$ & 68 & $0,08 \%$ \\
E.P.S.V. & 91 & $0,37 \%$ & 101 & $0,12 \%$ \\
S. garantía recíproca & 1 & $0,004 \%$ & 21 & $0,02 \%$ \\
Asoc. y fundaciones & 21.863 & $89,72 \%$ & 14.075 & $16,47 \%$ \\
\hline Total economía social & 24.368 & $\mathbf{1 0 0 , 0 0 \%}$ & $\mathbf{8 5 . 4 6 2}$ & $\mathbf{1 0 0 , 0 0 \%}$ \\
\hline
\end{tabular}

Fuente: elaboración propia a partir de OVES. Informe de Situación de la Economía Social Vasca 2015. 
El diferente comportamiento de los sectores está derivando en un importante proceso de recomposición interna de la Economía Social de Euskadi. Si del análisis se excluyen las asociaciones y las fundaciones y el foco se centra en los sectores que operan para el mercado, destaca por un lado, el importante desarrollo de los Centros Especiales de Empleo que entre el 2012 y el 2014 , crece en un 33\% el número de entidades hasta alcanzar el 9\% del empleo del conjunto de la Economía Social vasca, superando el peso específico de las Sociedades Laborales vascas.

Pero si el foco se centra en las Formas Clásicas de la Economía Social (FCES), esto es, las cooperativas y las Sociedades Laborales, encontramos que el proceso de recomposición resulta aún más significativo:

Desde el año 2004 hasta el 2014 el número de establecimientos cooperativos se incrementa de manera ininterrumpida, hasta alcanzar un incremento acumulado del $30 \%$. En cambio, las Sociedades Laborales, sea por quiebra o por reconversión jurídica, pierden el $32 \%$ de los establecimientos, siendo este proceso de declive especialmente significativo en el caso de las Sociedades Anónimas Laborales que pierden más de la mitad (el $54,7 \%$ ) de sus componentes en el espacio de una década.

\section{Tabla 2}

Evolución 2004-2014: establecimientos de cooperativas en relación a las FCES

\begin{tabular}{crrrrrrr}
\hline & 2004 & 2006 & 2008 & 2010 & 2012 & 2014 & $14 / 04$ \\
\hline Cooperativas & 1.717 & 1.855 & 1.895 & 2.029 & 2.194 & 2.233 & $30,05 \%$ \\
S.L & 1.315 & 1.241 & 1.091 & 968 & 884 & 894 & $-32,01 \%$ \\
S.A.L & 508 & 437 & 364 & 319 & 268 & 230 & $-54,72 \%$ \\
S.L.L & 807 & 804 & 727 & 649 & 616 & 664 & $-17,72 \%$ \\
\hline Total & 3.032 & 3.096 & 2.986 & 2.997 & 3.078 & 3.127 & $3,13 \%$ \\
\hline
\end{tabular}

Fuente: Elaboración propia a partir de Departamento de Empleo y Políticas Sociales del Gobierno Vasco. Estadística de la Economía Social.

El proceso de declive de las Sociedades Anónimas Laborales se refleja también en la evolución relativa al empleo ya que entre el 2008 y el 2014 pierden el $57 \%$ del empleo. El resultado es que en el 2014, por primera vez, las SALes presentan un volumen de empleo inferior al aportado por las Sociedades Laborales Limitadas (que con una reducción del 15\% del empleo muestran una mejor evolución que las SALes). 


\section{Tabla 3}

Evolución 2004-2014: empleo cooperativas en relación a las FCES y el conjunto de la CAPV

\begin{tabular}{|c|c|c|c|c|c|c|c|}
\hline & 2004 & 2006 & 2008 & 2010 & 2012 & 2014 & $14 / 08$ \\
\hline Total FCES & 60.733 & 63.073 & 62.616 & 59.915 & 55.809 & 54.581 & $-12,82 \%$ \\
\hline Coop. & 47.797 & 49.760 & 50.359 & 48.196 & 47.981 & 47.322 & $-6,03 \%$ \\
\hline S.A.L. & 8.563 & 8.238 & 7.451 & 7.355 & 4.005 & 3.177 & $-57,36 \%$ \\
\hline S.L.L. & 4.373 & 5.075 & 4.806 & 4.365 & 3.823 & 4.082 & $-15,06 \%$ \\
\hline Total CAPV & 929.200 & 954.200 & 986.100 & 937.800 & 898.400 & 892.200 & $-9,52 \%$ \\
\hline
\end{tabular}

Fuente: Elaboración propia a partir de Departamento de Empleo y Políticas Sociales del Gobierno Vasco. Estadística de la Economía Social.

Nota: los totales de la CAPV en base a la encuesta de población en relación con la actividad. Eustat.

En consecuencia, las cooperativas aumentan su peso relativo en el seno de las familias más representativas de la Economía Social vasca: Si al inicio del periodo de la crisis las cooperativas suponían el 56,6\% de los establecimientos, el 80,4\% del empleo, y el 85,6\% del VAB del conjunto de las FCES, en el 2014 las cooperativas representaban respectivamente el $71 \%$ de los establecimientos, el $86,7 \%$ del empleo y el $90,2 \%$ del VAB generado por el conjunto de las FCES.

La diferente evolución parece derivarse del mayor dinamismo de las cooperativas en la adaptación a las condiciones complejas del entorno. Los indicadores disponibles reflejan una mayor orientación hacia la innovación de las cooperativas (el $25,8 \%$ de las cooperativas realizaron algún tipo de actividad innovadora entre el 2012 y el 2014, frente al 17\% de las SALes y el $13,3 \%$ de las SLLs); un mayor esfuerzo en cuanto a inversiones materiales (las cooperativas presenten un ratio de 3.381 euros de inversión por empleado, frente al 2.866 euros de las SALes, y al 1.749 euros de las SLLs); y un comportamiento más dinámico de las cooperativas en cuanto a las exportaciones industriales (el 35,9\% de la facturación del 2014 de las cooperativas se corresponde con las exportaciones, frente al $27,7 \%$ de las SALes y el $9 \%$ de las SLLs).

Otro dato que parece dar muestra del mayor dinamismo de las cooperativas respecto a las sociedades laborales es el relativo a la estructura de edad de cada una de las familias de las FCES. Las sociedades laborales presentan una estructura de edad más envejecida que las cooperativas. Destaca 
el caso de las SALes donde casi la mitad (el 46\%) de las personas tenían más de 45 años en 2014, mientras que en las cooperativas era poco más de un tercio de las personas (el $35,8 \%$ ) la que sitúa en esa franja de edad.

\section{Tabla 4}

Comparativa cooperativas, sociedades anómimas laborales y sociedades limitadas laborales

\begin{tabular}{lccc}
\hline & Cooperativa & S.A.L & S.L.L. \\
\hline $\begin{array}{l}\text { Evolución del número de establecimientos } \\
(2004-2014)\end{array}$ & $30,05 \%$ & $-54,72 \%$ & $-17,72 \%$ \\
\hline $\begin{array}{l}\text { Evolución del volumen del empleo } \\
(2004-2014)\end{array}$ & $-6,03 \%$ & $-57,36 \%$ & $-15,06 \%$ \\
\hline $\begin{array}{l}\text { Evolución del Valor Ańadido Bruto } \\
\text { (2008-2014) }\end{array}$ & $-5,93 \%$ & $-52,67 \%$ & $-11,01 \%$ \\
\hline $\begin{array}{l}\text { \% empresas con actividades de innovación } \\
\text { (2012-2014) }\end{array}$ & $25,80 \%$ & $17,00 \%$ & $13,30 \%$ \\
\hline $\begin{array}{l}\text { Ratio de inversión en inmovilizado material } \\
(2014)\end{array}$ & 3.831 & 2.866 & 1.749 \\
\hline $\begin{array}{l}\text { Evolución de exportaciones industriales } \\
\text { (2008-2014) }\end{array}$ & $11,63 \%$ & $-16,17 \%$ & $102,92 \%$ \\
\hline $\begin{array}{l}\text { Peso exportaciones sobre facturación } \\
(2014)\end{array}$ & $35,90 \%$ & $27,70 \%$ & $9,00 \%$ \\
\hline $\begin{array}{l}\text { Porcentaje de trabajadores mayores de } \\
45 \text { ańos (2014) }\end{array}$ & $35,80 \%$ & $45,90 \%$ & $41,80 \%$ \\
\hline
\end{tabular}

Fuente: Elaboración propia a partir de Departamento de Empleo y Políticas Sociales del Gobierno Vasco. Estadística de la Economía Social 2014.

\subsection{La mayor capacidad de resiliencia de las cooperativas: las cooperativas manifiestan un mejor comportamiento en cuanto al mantenimiento de la actividad y el empleo en contextos de crisis}

El análisis comparado de los datos nos permite extraer una serie de conclusiones entorno a la resiliencia de las entidades de la Economía Social. Se ha entendido que las empresas vinculadas a este sector, comparativamente, muestran una mayor capacidad de resistencia en cuanto al man- 
tenimiento de la actividad y el empleo en contextos de crisis. A la luz de los datos presentados en el apartado anterior esta afirmación precisa de algunas matizaciones. La evolución del empleo durante la crisis refleja que el conjunto de las FCES han tenido un peor comportamiento respecto a la evolución general del empleo en la CAPV (las FCES pierden el 12,8\% del empleo frente al 9,5\% de la CAPV). Pero los datos permiten apreciar un comportamiento diferenciado según los sectores. Así, mientras que las Sociedades Laborales evolucionan peor que el conjunto de la economía de la CAPV, las cooperativas con un descenso del $6 \%$ del empleo en el periodo 2008-2014 demuestran un mejor comportamiento y, por lo tanto, mayor capacidad de resistencia al impacto de la crisis, tanto respecto a las Sociedades Laborales como al conjunto de la evolución del empleo de la CAPV.

La mayor capacidad de resiliencia de las cooperativas se confirma si la comparativa la realizamos con los modelos de empresa mercantiles. Los establecimientos cooperativos aumentan en un $17,8 \%$ aun en el período de crisis 2008-2014. Esta tendencia contrasta con la evolución negativa registrada por el conjunto del tejido empresarial vasco, que en el periodo analizado ve como desaparecen el 13,7\% de las empresas del territorio. A su vez, la evolución positiva de las cooperativas destaca frente a la evolución negativa de las Sociedades Limitadas (que pierden el 9,2\% de su tejido) y, sobre todo, de las Sociedades Anónimas ya que durante la crisis económica desaparecen más de un cuarto de las mismas (el 28\%).

\section{Tabla 5}

Evolución 2008-2014: total de empresas, sociedades anónimas y sociedades límitadas

\begin{tabular}{rrrrrc}
\hline & \multicolumn{1}{c}{$\mathbf{2 0 0 8}$} & \multicolumn{1}{c}{$\mathbf{2 0 1 0}$} & \multicolumn{1}{c}{2012} & \multicolumn{1}{c}{2014} & \multicolumn{1}{c}{$14 / 08$} \\
\hline Total empresas & 184.290 & 171.345 & 165.517 & 159.001 & $-13,72 \%$ \\
\hline Sociedad anónima & 8.964 & 8.194 & 7.215 & 6.453 & $-28,01 \%$ \\
Sociedad limitada & 46.534 & 47.349 & 44.189 & 42.246 & $-9,21 \%$ \\
\hline
\end{tabular}

Fuente: Eustat. Dirae.

En cuanto a la evolución del empleo durante el periodo 208-2014 el comportamiento de las cooperativas ha sido mejor tanto en relación a la evolución del empleo en el conjunto de la economía como en la comparativa con el resto de modelos mercantiles: 
Las cooperativas aun registrando una pérdida de seis puntos porcentuales, han manifestado un mejor comportamiento que la evolución del empleo en el conjunto de la economía de la CAPV, donde la destrucción del empleo según las diferentes fuentes estadísticas ha rondado entre el 9,5 y el $9,8 \%$. El comportamiento de las cooperativas ha sido mejor, también, tanto en la comparativa con las S.L. que pierden el 9,62\% del empleo que mantenían en el 2008 y, sobre todo, en comparación con las S.A. que padecen una destrucción del 20,5\% del empleo.

\section{Tabla 6}

Evolución 2008-2014: del empleo total CAPV, sociedad anónima y sociedad limitada

\begin{tabular}{cccccc}
\hline & 2008 & 2010 & 2012 & 2014 & $14 / 08$ \\
\hline Total empleo & 942.479 & 906.044 & 873.121 & 849.335 & $-9,88 \%$ \\
\hline S.A & 271.213 & 251.214 & 231.907 & 215.541 & $-20,52 \%$ \\
S.L & 279.755 & 277.586 & 262.059 & 252.845 & $-9,62 \%$ \\
\hline
\end{tabular}

Fuente: elaboración propia a partir de Eustat. Dirae.

La pauta se repite en el análisis de los sectores de la economía pero con algún matiz significativo. En todos los sectores el comportamiento del empleo en las cooperativas ha sido más positivo que la evolución que los sectores han mantenido en el conjunto de la CAPV y en comparación con las S.A donde el empleo se muestra más vulnerable sea cual sea el sector de referencia. Destaca, la mejor evolución de las cooperativas en la construcción $(-8,55 \%)$ frente a la negativa evolución del empleo en el conjunto de la CAPV $(-45,5 \%)$ y en las S.A $(-45,9 \%)$. En cuanto a la industria el empleo en las cooperativas $(-17,5 \%)$ ha evolucionado sensiblemente mejor que el empleo industrial de la CAPV (donde se pierde el $22 \%$ del empleo durante la crisis) y ostensiblemente mejor en comparación con las S.A. donde se destruye el 28,8\% del empleo desde el 2008. Los matices provienen del comportamiento sectorial del empleo en las Sociedades Limitadas: demuestra un mejor comportamiento que el empleo cooperativo en la industria $(-13,7 \%)$ y en los servicios (con un incremento del $5,2 \%$, ligeramente superior al crecimiento del $4,6 \%$ de las cooperativas), pero no en la construcción donde se destruye casi la mitad del empleo $(-49,3 \%)$ que existía en el 2008. 


\section{Tabla 7}

Evolución 2006-2014: de la estructura sectorial del empleo de cooperativas

\begin{tabular}{|c|c|c|c|c|c|c|}
\hline & 2006 & 2008 & 2010 & 2012 & 2014 & $14 / 08$ \\
\hline Primario & 98 & 190 & 154 & 101 & 156 & $-17,89 \%$ \\
\hline Industria & 24.525 & 23.329 & 20.897 & 20.917 & 19.238 & $-17,54 \%$ \\
\hline Construcción & 1.108 & 1.111 & 1.242 & 1.316 & 1.016 & $-8,55 \%$ \\
\hline Servicios & 24.028 & 25.729 & 25.903 & 25.647 & 26.914 & $4,60 \%$ \\
\hline Total & 49.760 & 50.359 & 48.196 & 47.981 & 47.322 & $-6,03 \%$ \\
\hline
\end{tabular}

Fuente: Elaboración propia a partir de Departamento de Empleo y Políticas Sociales del Gobierno Vasco. Estadística de la Economía Social.

\section{Tabla 8}

Evolución 2008-2014: personas empleadas por actividad (a10) y personalidad jurídica

\begin{tabular}{lccrrr}
\hline & 2008 & 2010 & 2012 & 2014 & \multicolumn{1}{c}{$14 / 08$} \\
\hline Total CAPV & & & & & \\
Primario & n.d. & n.d. & 11.910 & 12.968 & \\
Industria & 227.686 & 203.353 & 191.957 & 177.577 & $-22,00 \%$ \\
Construcción & 98.874 & 87.481 & 69.392 & 53.842 & $-45,54 \%$ \\
Servicios & 601.553 & 615.210 & 599.862 & 604.948 & $0,56 \%$ \\
\hline Sociedades anónimas & & & & & \\
Primario & n.d. & n.d. & 998 & 1.106 & \\
Industria & 118.664 & 101.813 & 94.518 & 84.453 & $-28,83 \%$ \\
Construcción & 22.983 & 20.520 & 17.017 & 12.435 & $-45,89 \%$ \\
Servicios & 128.401 & 128.881 & 119.374 & 117.547 & $-8,45 \%$ \\
\hline Sociedades limitadas & & & & & \\
Primario & n.d. & n.d. & 1.245 & 1.258 & \\
Industria & 72.725 & 69.281 & 66.303 & 62.742 & $-13,72 \%$ \\
Construcción & 45.831 & 42.464 & 31.617 & 23.232 & $-49,30 \%$ \\
Servicios & 157.458 & 165.841 & 162.894 & 165.613 & $5,18 \%$ \\
\hline
\end{tabular}

Fuente: elaboración propia a partir de Eustat. Dirae.

Nota: Las actividades industriales se presentan agregadas en industria, energía y saneamiento. 


\subsection{El reto de la industria avanzada: la contribución de las cooperativas es} más significativa en los segmentos estratégicos y tractores de la economía

El análisis del contexto económico revelaba la importancia que la industria, y más en concreto la industria manufacturera de alta tecnología, adquiere en la reorientación de la política económica europea. Se entiende que un sector industrial tecnológicamente avanzado es el sostén imprescindible en el nuevo escenario económico caracterizado por la pujanza de los actores emergentes, para el desarrollo de una economía sostenible con capacidad de crear empleos de alto valor ańadido. En este contexto, resulta pertinente que nos cuestionemos sobre la contribución que realiza el cooperativismo vasco al desarrollo de un sector industrial vasco tecnológicamente solvente.

El análisis de la evolución de la estructura sectorial del empleo detecta un relativo proceso de tercerización del tejido cooperativo. En términos de empleo, en el 2006 encontramos un reparto equitativo, con un ligero predominio del empleo industrial sobre los servicios (el 49,3\% del empleo se correspondía con la industria y el $48,3 \%$ con los servicios). Esta relación se invierte tras la crisis. En el 2014 el sector industrial representa el 40,7\% mientras que los servicios generan el 56\% del empleo cooperativo.

\section{Tabla 9}

Evolución 2006-2014: distribución relativa de la estructura sectorial empleo de cooperativas

\begin{tabular}{lrrrrrr}
\hline & $\mathbf{2 0 0 6}$ & $\mathbf{2 0 0 8}$ & $\mathbf{2 0 1 0}$ & $\mathbf{2 0 1 2}$ & $\mathbf{2 0 1 4}$ & $\mathbf{1 4 / 0 6}$ \\
\hline Primario & $0,2 \%$ & $0,4 \%$ & $0,3 \%$ & $0,2 \%$ & $0,3 \%$ & 0,1 \\
Industria & $49,3 \%$ & $46,3 \%$ & $43,4 \%$ & $43,6 \%$ & $40,7 \%$ & $-8,6$ \\
Construcción & $2,2 \%$ & $2,2 \%$ & $2,6 \%$ & $2,7 \%$ & $2,1 \%$ & $-0,1$ \\
Servicios & $48,3 \%$ & $51,1 \%$ & $53,7 \%$ & $53,5 \%$ & $56, \%$ & 7,7 \\
\hline Total & $\mathbf{1 0 0 \%}$ & $\mathbf{1 0 0} \%$ & $\mathbf{1 0 0} \%$ & $\mathbf{1 0 0} \%$ & $\mathbf{1 0 0} \%$ & \\
\hline
\end{tabular}

Fuente: Elaboración propia a partir de Departamento de Empleo y Políticas Sociales del Gobierno Vasco. Estadística de la Economía Social.

Esta tendencia se aprecia también en la evolución de la distribución sectorial del Valor Añadido Bruto generado por las cooperativas: las cooperativas industriales en el periodo 2008-2014 reducen su participación en 
el VAB del 55,5\% al 49,2\%. A su vez, el sector servicios aumenta su peso pasando a representar del 42,7\% al 49,4\%. En el 2014 la participación de ambos sectores en el VAB generado muestra una distribución equilibrada.

\section{Tabla 10}

Evolución 2008-2014: de la distribución del VAB sectorial de las cooperativas

\begin{tabular}{l|rr|rr|rr|c}
\hline & \multicolumn{2}{|c|}{2008} & \multicolumn{2}{|c|}{2012} & \multicolumn{2}{|c|}{2014} & \multirow{2}{*}{$\begin{array}{c}\text { 14/08 } \\
\text { (c.a) }\end{array}$} \\
\cline { 2 - 7 } & \multicolumn{1}{|c|}{ ABS. } & \%VER. & ABS. & \%VER. & ABS. & \%VER. & \multicolumn{1}{c}{ AB. } \\
\hline Primario & 5.388 .407 & 0,2 & 1.443 .557 & 0,1 & 3.520 .274 & 0,1 & $-34,67 \%$ \\
Industria & 1.391 .499 .961 & 55,5 & 1.196 .577 .748 & 50,0 & 1.159 .781 .571 & 49,2 & $-16,52 \%$ \\
Construcción & 38.866 .872 & 1,5 & 39.203 .323 & 1,6 & 29.544 .243 & 1,3 & $-23,99 \%$ \\
Servicios & 1.071 .847 .671 & 42,7 & 1.157 .087 .983 & 48,3 & 1.166 .038 .867 & 49,4 & $8,79 \%$ \\
\hline Total & 2.507 .602 .910 & 100,0 & 2.394 .312 .612 & 100,0 & 2.358 .884 .955 & 100,0 & $-5,93 \%$ \\
\hline
\end{tabular}

Fuente: Elaboración propia a partir de Departamento de Empleo y Políticas Sociales del Gobierno Vasco. Estadística de la Economía Social.

No obstante, aún es pronto para determinar si nos encontramos ante lo que puede ser una tendencia estructural hacia la tercerización de la economía cooperativa en Euskadi o si, por el contrario, no es más que el reflejo de un movimiento cíclico fruto del diferente comportamiento de los sectores a la coyuntura del periodo de crisis y, por lo tanto, modificable por un contexto de recuperación del pulso por parte de las cooperativas industriales.

Esta evolución no distorsiona la relativa especialización industrial por la que se caracteriza el cooperativismo vasco, ni la aportación específica que realizan las cooperativas a la especialización industrial de la economía vasca. Esta contribución se hace más evidente si enfocamos el análisis en las empresas tractoras del territorio. Para ello proponemos centrar la mirada en las grandes empresas de la industria manufacturera vasca. El ámbito de la industria manufacturera, como ya ha sido señalado en el texto, por el determinante rol que asume en la tracción de las economías en los nuevos escenarios globales y complejos; y las empresas grandes, por la relevancia que asume la dimensión de las empresas en los contextos económicos que venimos analizando.

Los datos para la base del análisis son las que dispone el Eustat en el Directorio de Actividades Económicas. La dificultad que encontramos es que para este nivel estadístico Eustat no presenta los datos desagregados 
de las cooperativas, sino que éstos aparecen subsumidos bajo la categoría «Otras sociedades». No obstante, dado el peso específico que asumen las cooperativas en los campos de la industria y, específicamente, en el ámbito de las grandes empresas, para este ámbito concreto del objeto del análisis, pensamos que resulta factible identificar la categoría de "Otras Sociedades» con la incidencia específica de las empresas cooperativas del territorio.

En esta línea, en el cuadro que presentamos a continuación se observa que la contribución del cooperativismo al tejido de las empresas tractoras del territorio es más notoria según avancemos en la escala de la dimensión de las empresas. En la franja de empresas de 100 a 249 trabajadores las cooperativas junto con las otras empresas agrupadas bajo la categoría de «Otras Sociedades» suponen cerca del $11 \%$ del tejido empresarial, mientras el resto se corresponde con una mayoría de S.A.s (el 56,6\%) y un tercio de S.L.s (32,5\%). En la siguiente franja, la de las empresas de 250 a 499 trabajadores, la proporción de cooperativas aumenta, ya que las «Otras sociedades» constituyen el $28,5 \%$ del tejido empresarial de esta franja, superando en número y proporción a las Sociedades Limitadas (un 21,42\%), mientras que las Sociedades Anónimas suponen la mitad (el 50\%) de las empresas de esta franja. Pero el dato más significativo lo encontramos en la franja de las empresas más grandes. Se aprecia que entre las 27 empresas mayores de 500 trabajadores que se encuentran en el sector de la industria manufacturera de nuestro país 11 (el 40,7\%) son Sociedades Anónimas, 10 (el $37 \%$ ) son cooperativas, y 6 (el 22,2\%) se corresponden con las Sociedades Limitadas.

\section{Tabla 11}

Empresas en la industria manufacturera según dimensión y personalidad jurídica. 2016

\begin{tabular}{l|rr|rr|rr}
\hline & \multicolumn{2}{|c|}{ De 100 a 249} & \multicolumn{2}{c|}{ De 250 a 499 } & \multicolumn{2}{c}{$>=500$} \\
\cline { 2 - 8 } & ABS. & \%VER. & ABS. & \%VER. & ABS. & \%VER. \\
\hline S.A. & 94 & $56,63 \%$ & 21 & $50,00 \%$ & 11 & $40,70 \%$ \\
S.L. & 54 & $32,53 \%$ & 9 & $21,42 \%$ & 6 & $22,22 \%$ \\
Otras sociedades & 18 & $10,84 \%$ & 12 & $28,57 \%$ & 10 & $37,03 \%$ \\
\hline Total CAPV & 166 & $100,00 \%$ & 42 & $100,00 \%$ & 27 & $100,00 \%$ \\
\hline
\end{tabular}

Fuente: elaboración propia a partir de Eustat. Dirae.

Nota: empresas con sede social en la CAPV. 
3.4. Las cooperativas demuestran una mayor orientación innovadora que el resto de empresas

Las evidencias apuntan a una mayor orientación de las cooperativas hacia las actividades de innovación. El dato cobra relevancia dada la importancia que adquieren los sistemas locales de innovación en el contexto del nuevo ciclo económico. Hoy prácticamente nadie discute el hecho de que la innovación y el desarrollo tecnológico son factores determinantes para garantizar la competitividad y, por tanto, la viabilidad de las empresas en estos escenarios globales y complejos. Es más, cada vez es más compartida la idea de que el esfuerzo innovador va a adquirir una importancia aún más determinante en esta nueva era de disrupciones tecnológicas a la que parecemos abocados.

Los datos disponibles señalan que en el periodo 2012-2014 el porcentaje de cooperativas que desarrollaron actividades relacionadas con la innovación (el 25,8\% de cooperativas) es mayor que el porcentaje del conjunto de las empresas de la CAPV (el 17\% de las empresas realizaron actividades relacionadas con la innovación).

\section{Gráfico 1}

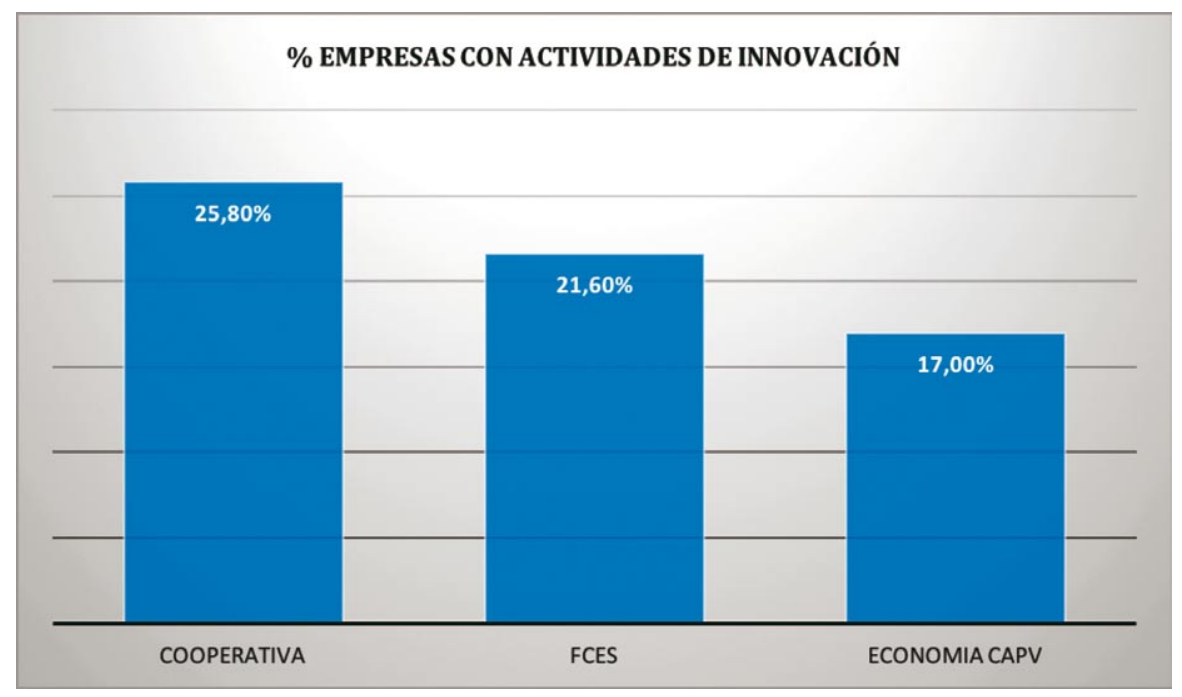

Fuente: Elaboración propia a partir de Departamento de Empleo y Políticas Sociales del Gobierno Vasco. Estadística de la Economía Social 2014.

La mayor orientación innovadora de las cooperativas respecto al resto de empresas se confirma en el Informe de Competitividad del País Vasco del 2015 elaborado por el Instituto Orkestra con datos del 2013. 
Tabla 12

Esfuerzo en innovación. Comparativa cooperativas y resto de empresas $\left(^{*}\right)$ (referencia 2013)

\begin{tabular}{|c|c|c|c|}
\hline & Cooperativa & $\begin{array}{l}\text { Resto de } \\
\text { empresas }\end{array}$ & $\begin{array}{c}\text { Total } \\
\text { empresas }\end{array}$ \\
\hline $\begin{array}{l}\text { Empresas que cooperan } \\
\text { (\% respecto al total de empresas) }\end{array}$ & 28,1 & 10,1 & 11,0 \\
\hline $\begin{array}{l}\text { Gastos en innovación } \\
\text { (\% respecto de las ventas) }\end{array}$ & 3,9 & 2,0 & 2,1 \\
\hline $\begin{array}{l}\text { Gasto en maquinaria } \\
\text { (\% respecto a gasto en innovación) }\end{array}$ & 14,0 & 10,2 & 19,2 \\
\hline $\begin{array}{l}\text { Gasto } \mathrm{I}+\mathrm{D} \text { externa } \\
\text { (\% respecto a gasto en innovación) }\end{array}$ & 17,0 & 22,6 & 19,0 \\
\hline $\begin{array}{l}\text { Empresas innovadoras } \\
\text { (\% respecto al total de empresas) }\end{array}$ & 52,6 & 31,9 & 33,1 \\
\hline $\begin{array}{l}\text { Innovación no tecnológica } \\
\text { (\% respecto a total de empresas) }\end{array}$ & 47,7 & 23,8 & 25,3 \\
\hline $\begin{array}{l}\text { Innovación tecnológica y no tecnológica } \\
\text { (\% respecto a total de empresas })\end{array}$ & 16,3 & 11,8 & 12,1 \\
\hline $\begin{array}{l}\text { Innovación de producto } \\
\text { (\% respecto a total de empresas) }\end{array}$ & 36,9 & 17,9 & 19,1 \\
\hline $\begin{array}{l}\text { Exportación } \\
\text { (\% respecto a facturación) }\end{array}$ & 46,4 & 20,7 & 25,8 \\
\hline $\begin{array}{l}\text { Ventas nuevas para la empresa } \\
\text { (\% respecto a las ventas totales) }\end{array}$ & 16,8 & 9,0 & 11,0 \\
\hline $\begin{array}{l}\text { Ventas nuevas para el mercado } \\
\text { (\% respecto a las ventas totales) }\end{array}$ & 6,5 & 2,7 & 3,3 \\
\hline $\begin{array}{l}\text { Coste laboral por empleado } \\
\text { (miles de euros) }\end{array}$ & 58,0 & 35,0 & n.d \\
\hline $\begin{array}{l}\text { Endeudamiento } \\
(\%)\end{array}$ & 56,7 & n.d & 49,6 \\
\hline
\end{tabular}

Fuente: Orkestra. Informe de competitividad 2015.

${ }^{*}$ Excepto empresas con capital extranjero. 
Según el estudio las cooperativas en el ámbito de la innovación destacan respecto al resto de empresas por:

- Su mayor grado de cooperación en actividades y procesos de innovación entre empresas.

- Un mayor esfuerzo en innovación, que medido en base al porcentaje de gasto respecto al total de las ventas es casi el doble que el que destinan el resto de empresas.

- Una mayor generación de capacidades internas para el desarrollo de las actividades de innovación.

- Un mayor porcentaje de empresas innovadoras. El promedio de establecimientos innovadores entre las cooperativas (el 52,6\%) era superior tanto al promedio de la CAPV (el 33,1\%), como al porcentaje del resto de empresas (el 31,9\%). Orkestra destaca los datos del ámbito industrial donde el porcentaje de cooperativas innovadoras alcanzaba al $71,3 \%$. Este dato destaca por su singularidad ya que si durante la crisis, en la mayor parte de los países y tipos de empresas tuvo lugar una disminución del porcentaje de empresas innovadoras, esto no ocurrió en el caso de las cooperativas vascas.

- Un desempeño innovador superior al resto de empresas de la CAPV, tal y como indican la mayor innovación de producto, la innovación no tecnológica y el mayor porcentaje de cooperativas que combinan las innovaciones de carácter tecnológico y no tecnológico.

-Y, por último, un mejor comportamiento en cuanto al grado de novedad de los productos comercializados, ya sea en cuanto al porcentaje de ventas nuevas para la empresa, como en el caso de las ventas nuevas para el mercado.

Lo significativo es que esta mayor orientación innovadora y el mayor esfuerzo en el desarrollo de actividades de $\mathrm{I}+\mathrm{D}$ de las cooperativas se realiza incluso en un contexto de recesión económica y de reducción de resultados y márgenes comerciales de las cooperativas. La evolución que los resultados económicos de las cooperativas sufren durante el periodo de crisis no deja lugar a dudas. Los beneficios globales de las cooperativas en relación al 2006 se reducen en casi un 84\%, siendo la reducción de resultados en el caso de las cooperativas industriales del $64,8 \%$.

El propio informe de Orkestra destaca que el notable esfuerzo innovador de las cooperativas se estaba realizando en un contexto en el que, en cuanto a ventas, la evolución de las cooperativas no era mejor que el resultado que estaban obteniendo el resto de empresas, y en el que en términos de activos financieros el balance de las cooperativas presentaba una situación aún peor (Orkestra, 2015). 


\section{Tabla 13}

Evolución 2006-2014: de los resultados económicos en las cooperativas (c.a. en precios corrientes)

\begin{tabular}{lrrrrrr}
\hline & \multicolumn{1}{c}{2006} & \multicolumn{1}{c}{2008} & \multicolumn{1}{c}{2010} & \multicolumn{1}{c}{2012} & \multicolumn{1}{c}{2014} & \multicolumn{1}{c}{$14 / 06$} \\
\hline Primario & 460.777 & 509.643 & 183.262 & -154.517 & 242.466 & $-47,38 \%$ \\
Industria & 260.588 .440 & 122.996 .415 & 101.536 .126 & 115.110 .035 & 91.566 .703 & $-64,86 \%$ \\
Construcción & 4.760 .802 & 1.944 .301 & 2.094 .433 & -2.894 .391 & -15.682 & $-100,32 \%$ \\
Servicios & 235.996 .947 & 216.001 .261 & 109.006 .466 & -27.012 .571 & -11.059 .582 & $-104,68 \%$ \\
\hline Total & 501.806 .966 & 341.451 .620 & 212.820 .287 & 85.048 .557 & 80.733 .905 & $-83,91 \%$ \\
\hline
\end{tabular}

Fuente: elaboración propia a partir de Departamento de Empleo y Políticas Sociales del Gobierno Vasco. Estadística de la Economía Social.

Esta realidad alimenta una serie de dudas respecto a la disparidad entre el esfuerzo innovador y los resultados económicos que generan las cooperativas. Pero pensamos que del análisis de los datos se puede concluir que el esfuerzo en innovación no deja de ser una prioridad en las cooperativas a pesar de los contextos de crisis y la reducción de los márgenes de los resultados económicos. Y ello, seguramente, porque se entiende que la apuesta por la innovación y el desarrollo tecnológico es la única forma de adaptar la empresa a las nuevas exigencias del mercado, garantizar la viabilidad y la sostenibilidad del proyecto empresarial cooperativo, y poder mantener, a través de la incorporación de mayor valor añadido, las cantidades y las cualidades del empleo cooperativo.

\section{Conclusiones}

El presente trabajo presenta una serie de evidencias respecto a la aportación que las cooperativas vascas realizan a los retos que el tejido productivo del territorio enfrenta en el nuevo ciclo económico. Las evidencias se sostienen en el análisis de las fuentes estadísticas publicadas por diferentes agencias e instituciones. Pensamos que el valor del presente trabajo se encuentra en el estudio sistemático de las posibilidades analíticas que es posible extraer de las fuentes de información públicas. Pero es preciso señalar las limitaciones encontradas en esta vía: la disparidad de datos según las diferentes fuentes estadísticas, y la no disponibilidad de datos desagregados en función de la entidad jurídica de las empresas en variables significativas, son algunas de las dificultades que nos hemos encontrado en el camino. 
Es por ello que hablamos de conclusiones provisionales. Entendemos que se precisa el acceso a nuevas fuentes de información en algunos indicadores significativos para afinar el análisis y poder extraer conclusiones aún más sólidas.

Las limitaciones estadísticas no desvirtúan los indicios encontrados en esta investigación respecto las aportaciones que realizan las cooperativas en relación a los retos que enfrenta el tejido productivo vasco. Nos gustaría terminar el estudio resaltando las siguientes ideas:

a) Las cooperativas, en líneas generales, presentan un mejor comportamiento que el resto de las empresas en la evolución de las principales variables económicas. El mejor comportamiento de las cooperativas se da tanto en relación a las Sociedades Laborales, como a las Sociedades Anónimas y las Sociedades Limitadas. Esto parece derivarse del mayor dinamismo de las cooperativas en la adaptación a las condiciones complejas del entorno. Las cooperativas no sólo mantienen mejor el empleo y la actividad, sino que hacen un mayor esfuerzo en su adaptación a las exigencias económicas del entorno tal y como reflejan la mayor orientación innovadora y la mayor orientación exportadora de las cooperativas respecto al resto de empresas. De ello, se pueden derivar una serie de importantes consideraciones:

- Las cooperativas, superando la lectura tradicional, no sólo aportan capacidad de resiliencia respecto a la actividad y el empleo, sino que aportan también un mayor dinamismo a las características del tejido productivo de la CAPV. Se podría argumentar, además, que si las cooperativas son más resilientes es también por su mayor orientación inversora en el desarrollo humano, organizativo y tecnológico que permiten una mejor adaptación a las condiciones cambiantes del contexto.

- Si la crisis de Fagor Electrodomésticos alimentó dudas respecto a la eficiencia del modelo cooperativo para enfrentar el escenario complejo de la globalización, el presente estudio despeja las dudas sobre la eficiencia de las cooperativas. Al contrario, los indicios apuntan a una mayor eficiencia de las cooperativas en comparación al resto de modelos de empresa.

- Pensamos que todo ello tiene derivadas en cuanto al ámbito de las políticas públicas en relación al objeto de impulsar la participación de las personas en el ámbito de la empresa. Como se demuestra en el caso de las cooperativas, una mayor participación de las personas en la empresa repercute en los objetivos de enraizar las empresas al territorio, reforzar los mecanismos de 
cohesión social y de posicionar mejor al tejido productivo del territorio en el escenario económico global y complejo que toca enfrentar.

b) El incremento del tejido cooperativo en un contexto de crisis y reducción de actividad empresarial y económica es un dato significativo en sí. El mayor dinamismo en este periodo se ha dado entre las pequeñas cooperativas más vinculadas al sector servicios. Ello abre la hipótesis de si el mayor dinamismo del cooperativismo no se sostiene también en la conexión del modelo con las nuevas aspiraciones y perspectivas culturales que parecen emerger en la sociedad respecto al trabajo. De confirmarse la hipótesis se reforzaría en este escenario el impulso al proyecto histórico de vincular la eficiencia de las organizaciones empresariales con los procesos de participación y humanización del trabajo en el desarrollo de un nuevo paradigma empresarial.

c) Las cooperativas aportan capacidad innovadora al sistema local de innovación vasco. Las evidencias estadísticas confirman una mayor orientación de las cooperativas hacia las actividades de innovación. Lo significativo es que esta mayor orientación innovadora y el mayor esfuerzo en el desarrollo de actividades de $\mathrm{I}+\mathrm{D}$ de las cooperativas se realiza incluso en un contexto de recesión económica y de reducción de los resultados comerciales de las cooperativas. Es más, los indicios apuntan a que el esfuerzo innovador de las cooperativas se realiza en un contexto en el que, en cuanto a ventas, la evolución de las cooperativas no era mejor que el resultado que estaban obteniendo el resto de empresas, y en el que en términos de activos financieros el balance de las cooperativas presentaba una situación aún peor. De ello se pueden derivar dos consideraciones:

- Las cooperativas parecen demostrar una orientación innata hacia la innovación. Parece que existen una serie de valores o factores del modelo cooperativo que inciden en esa dirección. La realidad es que las cooperativas realizan el esfuerzo inversor en innovación aún en contextos de recesión, de reducción de beneficios y de sacrificios personales (bajada de anticipos, reubicaciones). Pensamos que ello guarda una estrecha relación con la vocación social de las cooperativas: la apuesta por la innovación y el desarrollo tecnológico se entiende que es la única forma de adaptar la empresa a las nuevas exigencias del mercado, garantizar la viabilidad y la sostenibilidad del proyecto empresarial cooperativo, y poder mantener, a través de la capacidad de generación de mayor valor añadido, el volumen y las cualidades del empleo cooperativo. 
- Ahora bien, las cooperativas también necesitan de capital para el desarrollo de sus proyectos empresariales. Las dudas surgen al reflexionar si todo ello no es posible si no es a costa de las rentabilidades y de un mayor endeudamiento de las cooperativas. Las comparativas con los resultados, sobre todo, de las empresas del territorio con capitales extranjeros apuntan en esa dirección, lo cual podría poner en cuestión la competitividad futura de los proyectos cooperativos.

d) El estudio identifica que la aportación de las cooperativas adquiere una mayor relevancia en los segmentos estratégicos y tractores de la economía. El análisis del contexto económico revela la importancia que la industria, y más en concreto la industria manufacturera de alta tecnología, adquiere en la reorientación de la política económica europea. Se entiende que un sector industrial tecnológicamente avanzado es el sostén imprescindible en el nuevo escenario económico caracterizado por la pujanza de los actores emergentes, para el desarrollo de una economía sostenible con capacidad de crear empleos de alto valor añadido. La constatación del peso específico de las cooperativas entre las grandes industrias tractoras del territorio, junto con el importante esfuerzo en innovación y desarrollo que desarrollan estas empresas nos llevan a afirmar que las cooperativas están coliderando el proceso de transición de la economía vasca hacia un modelo de desarrollo sostenido sobre la base de un tejido industrial tecnológicamente avanzado.

e) El peso específico que el cooperativismo asume entre las grandes empresas tractoras del territorio abre la puerta a nuevas hipótesis respecto al impacto de este modelo en la sociedad. Indicios de este potencial impacto se detectan al observar, por ejemplo, el diferente comportamiento de los territorios de la CAPV en los indicadores de innovación. Gipuzkoa, territorio donde el tejido cooperativo industrial es significativamente más denso, muestra unos porcentajes de Gasto en I+D (el 2,25\% del PIB en 2015) que superan con claridad el promedio europeo, mientras que Bizkaia $(1,73 \%)$ y Araba $(1,48 \%)$ muestran unos valores más modestos. Es más, como ya se ha analizado en el estudio, en el 2014 las empresas guipuzcoanas destinaron a labores de $\mathrm{I}+\mathrm{D}$ un total de 423,6 millones de euros, lo cual suponía superar los 412,5 millones de las empresas de Bizkaia, la provincia más poblada del territorio.

Nos preguntamos si parecido impacto se podría observar en los indicadores relativos a la aspiración del cooperativismo por construir una sociedad más justa y equitativa. Los estudios académicos del 
ámbito de las desigualdades han evidenciado el proceso de incremento de desigualdades y de concentración de la riqueza que padecen las sociedades económicamente avanzadas desde la década de los 80 del siglo Xx. Esto se está dando por dos vías principalmente: por los notables aumentos salariales de determinados segmentos profesionales y por los procesos de acumulación de capital que se producen en el contexto de la desregulación de los mercados. Las cooperativas se caracterizan por subordinar el capital a la primacía del trabajo y por fijar un estrecho intervalo en los ingresos por trabajo. ¿El hecho de que entre las grandes empresas tractoras de Gipuzkoa predominen las cooperativas, deriva en una estructura más igualitaria de la sociedad guipuzcoana en relación al resto de territorios? Es ésta una cuestión que merecería la pena explorar.

f) La capacidad del cooperativismo para conjugar el desarrollo económico con unos criterios más distribuidos e inclusivos, es un factor impulsor en la configuración del territorio vasco como un Sistema Local de Innovación. Analizábamos cómo el conocimiento, adherido al territorio, es uno de los factores determinantes de la competitividad basada en la innovación, y que la eficiencia económica del conocimiento depende mucho de la calidad de su flujo y del nivel de transferencia entre los diferentes agentes del sistema. En este sentido, el desarrollo de una estrategia innovadora será más eficiente si consigue la movilización y la implicación de los agentes plurales del territorio y para ello resulta determinante la configuración de un proyecto común de desarrollo. Conciliar las estrategias competitivas con los objetivos de la cohesión social y la integración socioeconómica resulta vital en este escenario. Las cooperativas por su capacidad económica y empresarial, y por sus rasgos innatos por la generación de un modelo de desarrollo distribuido parecen destinadas a asumir un rol estratégico en el nuevo ciclo económico que enfrenta nuestro país.

\section{Bibliografía}

Astigarraga, E. (2016): Escenarios para Mondragon Unibertsitatea al 2025. Tesis doctoral no publicada.

Azurmendi, J. (1984): El hombre cooperativo. Pensamiento de Arizmendiarreta, Euskadiko Kutxa, Arrasate.

Borja, A. (2012): «Euskal estatuari begira: Ahuleziak, indarrak eta krisi ekonomikoaren ondorioak euskal ekonomia-ehunean» en VVAA Euskal estatuari bidea zabaltzen. Lurraldea eta sozioekonomia, Ipar-Hegoa Fundazioa, Bilbo. 
Consejo Económico y Social Vasco (2015): Dinamismo empresarial, creación de riqueza y empleo: Un análisis comparado desde la CAPV, Estudios e informes N. 13, CES.

Duch, N. y Blanco, R. (2016): «Estructuras productivas europeas» en CostaCampi, M.T.; Garrido, A.; Parellada, M. y Sanromà, E. (eds.): Economía europea. Crecimiento, competitividad y mercados, Alianza editorial, Madrid.

García Delgado, J.L. y Myro, R. (2016): «El crecimiento económico en Europa» en Costa-Campi, M.T.; Garrido, A.; Parellada, M. y Sanromà, E. (eds.): Economía europea. Crecimiento, competitividad y mercados, Alianza editorial, Madrid.

García Quevedo, J. (2016): "Capital tecnológico y sistemas de innovación europeos» en Costa-Campi, M.T.; Garrido, A.; Parellada, M. y Sanromà, E. (eds.): Economía europea. Crecimiento, competitividad y mercados, Alianza editorial, Madrid.

B+I Strategy (2016): Dossier sobre la participación de las personas en la empresa, Unpublished manuscript, Gipuzkoako Foru Aldundia.

Kamp, B. (2016): «Servitización: Génesis, temas actuales y mirada al futuro, Ekonomiaz, n. ${ }^{\circ} 89$, págs. 252-279.

Larrańaga, J. (1998): El cooperativismo de Mondragón. Interioridades de una utopía, Otalora, Aretxabaleta.

Letta, E. (2017): Hacer Europa y no la guerra. Una apuesta europeísta frente a Trump y el Brexit, Península, Barcelona.

Molinas, C. y Ramírez, F. (2017): La crisis existencial de Europa, Deusto, Barcelona.

Navarro, M. (2010): «Retos para el País Vasco, tras tres décadas de desarrollo del sistema y de las políticas de innovación», Ekonomiaz, n.o 25, págs. 136-183.

Navarro, M. y Sabalza, X. (2016): «Reflexiones sobre la industria 4.0 desde el caso vasco", Ekonomiaz, n. ${ }^{\circ}$ 89, págs. 142-173.

Orkestra (2015): Informe de competitividad del País Vasco 2015. Transformación productiva en la práctica, Fundación Deusto, Bilbao.

Orkestra (2017): Informe de competitividad del País Vasco 2017 ¿Y mañana?, Fundación Deusto, Bilbao.

Ormaetxea, J.M. (1999): Orígenes y claves del cooperativismo de Mondragón, Euskadiko Kutxa-Saiolan, Arrasate.

Ormaetxea, J.M. (2003): Didáctica de una experiencia empresarial, Euskadiko Kutxa-Saiolan, Arrasate.

Ortega, A. (2016): La imparable marcha de los robots, Alianza editorial, Madrid.

OVES (2016): Informe de situación de la Economía Social Vasca. Año 2015, Oves/Geeb, Donostia.

Pérez, C. (2010): «Revoluciones tecnológicas y paradigmas tecno-económicos, Cambridge Journal of Economics, Vol. 1, n.o 34, págs. 185-202.

PROSPEKTIKER (2012): Estudio Delphi sobre el futuro de las organizaciones: Cooperativismo y otros modelos, Mondragón Corporación, Arrasate. 
Sudrià, C. y Garrido, A. (2016): «La economía europea desde 1950 hasta la actualidad» en Costa-Campi, M.T.; Garrido, A.; Parellada, M. y Sanromà, E. (eds.): Economía europea. Crecimiento, competitividad y mercados, Alianza editorial, Madrid.

Ugarte, J. (2013): España está en crisis. El mundo no. Deusto, Barcelona.

Zelaia, A. (2015): Euskal ekonomiaren egiturazko erronkak, Ekai Center, Arrasate.

Zurbano, M. (2005): «Las regiones como sujetos de la economía global. Euskadi en la gobernanza multidimensional», Ekonomiaz, n.o 58, págs. 196-231. 